\title{
Uso de los servicios de redes sociales por la generación $\mathbf{Y}$
}

\author{
Use of social networking services by the generation $Y$ \\ Patricio Ramírez-Correa $^{1} \quad$ Jorge Arenas-Gaitán ${ }^{2} \quad$ Javier Rondan-Cataluña $^{2}$ \\ Recibido 27 de diciembre de 2011, aceptado 24 de septiembre de 2012 \\ Received: December 27, 2011 Accepted: September 24, 2012
}

\begin{abstract}
RESUMEN
El propósito de este artículo es estudiar la adopción de los Servicios de Redes Sociales (SRS) por parte de integrantes de la generación Y. Se presenta la validación empírica de un modelo teórico que explica el uso de los SRS como resultado de antecedentes individuales que afectan la percepción de su utilidad. El resultado del trabajo de campo realizado con una muestra de 771 usuarios de SRS fue analizado en un modelo de ecuaciones estructurales basado en PLS (Partial Least Squares). El análisis de estos datos soporta el modelo teórico que explica el uso de SRS en función de la percepción de su facilidad de uso y de la percepción de su utilidad. Adicionalmente, el modelo explica que la percepción de su utilidad es consecuencia de la percepción del grado de identidad social, altruismo y telepresencia.
\end{abstract}

Palabras clave: Servicios de redes sociales, aceptación de tecnología, generación Y, identidad social, altruismo, telepresencia.

\begin{abstract}
The purpose of this paper is to study the adoption of Social Networking Services (SNS) by members of generation $Y$. The paper shows an empirical validate of a theoretical model that explains the use of SNS as a result of individual antecedents that affect the perception of its usefulness. The result of the fieldwork with a sample of 771 SNS users was analyzed in a structural equation model based on PLS (Partial Least Squares). The analysis of this data supports the theoretical model that explains the use of SNS in terms of perceived ease of use and perceived usefulness. Additionally, the model explains that the perception of usefulness is due to the perception of the degree of social identity, altruism, and telepresence.
\end{abstract}

Keywords: Social networking services, technology acceptance, generation Y, social identity, altruism, telepresence.

\section{INTRODUCCIÓN}

La generación Y es considerada un grupo de vanguardia en el uso de tecnologías de la información [1]. Por tanto, saber cómo esta generación adopta y usa los nuevos servicios de la Web 2.0 es relevante para analizar el futuro desarrollo de estas tecnologías. En este estudio tratamos de aportar en ese sentido.
La Web 2.0 es un fenómeno emergente y de gran interés para el estudio científico. En contraste con los sitios de la Web 1.0, cuyo contenido es generado por un proveedor, en los sitios de la Web 2.0 el contenido es generado por el usuario. Es en la Web 2.0 donde ha habido una explosión dramática en prosumición. La prosumición incluye tanto la producción como el consumo de contenido. Se puede argumentar que la

\footnotetext{
1 Escuela de Ciencias Empresariales. Universidad Católica del Norte. Larrondo 1281. Coquimbo, Chile. E-mail: patricio.ramirez@ucn.cl

2 Departamento de Administración de Empresas y Marketing. Universidad de Sevilla. Ramón y Cajal 1. Sevilla, España. E-mail: jarenas@us.es; rondan@us.es
} 
Web 2.0 debe ser vista como crucial en el desarrollo de los "medios de prosumo". La Web 2.0 facilita la implosión de la producción y el consumo [2].

En este contexto, la popularidad de los Servicios de Redes Sociales (SRS) es un fenómeno sin discusión. Según datos de Nielson Wire, los sitios de SRS (tales como Facebook, Twitter o Tuenti) son visitados por tres cuartas partes de los consumidores que ingresan a Internet [3]. De hecho, Facebook da servicios a más de 500 millones de usuarios activos y estos gastan en total sobre 700 billones de minutos al mes en su sitio [4]. Y esta popularidad está en aumento, el año 2010 el visitante promedio gasto 66\% más tiempo en estos sitios que en el año 2009 [3].

El núcleo de un sitio SRS es una colección de perfiles de usuario, donde los miembros registrados pueden colocar información que desean compartir con los demás. En su mayor parte, los usuarios participan en dos tipos de actividades en el sitio: Como proveedor, al crear nuevos contenidos mediante la edición de sus perfiles (por ejemplo, agregar imágenes, subir música, escribir blogs y mensajes), o como consumidor, utilizando contenido que otros crean (por ejemplo, viendo fotos, descargando música, leyendo blogs y mensajes). En la mayoría de los sitios SRS los usuarios pueden agregar otros usuarios a sus redes de "amigos" o contactos. Por lo general, un usuario inicia la invitación, y el otro usuario acepta o la rechaza. Cuando se aceptan, los dos perfiles se vinculan [5].

Otro fenómeno actual de singular interés para el estudio académico es recogido por la disciplina del marketing con el nombre de generación Y. La generación Y representa a las personas que nacieron entre 1978 y 1994 [6]. Diversos estudios concuerdan en que los integrantes de esta generación son conocedores de la tecnología y son usuarios del comercio electrónico a través de Internet [1].

El propósito general de este trabajo es estudiar la adopción de los SRS por parte de integrantes de la generación Y. En particular, el objetivo del estudio es validar empíricamente un modelo teórico que explica el uso de los SRS como resultado de antecedentes individuales que afectan la percepción de su utilidad.

\section{REVISIÓN DE LA LITERATURA}

\section{Generación Y}

El estudio de mercados ha utilizado la teoría de las generaciones para el análisis de los consumidores a través de la caracterización de las generaciones identificadas a partir de 1900 y basada en cohortes de unos 20 años, a saber, G1, Silenciosa, generación Baby Boomers, generación X, generación Y, y para los nacidos del 2003 y en adelante, generación Z. Cada generación es estereotipada con una serie de características actitudinales y de comportamiento, predominantemente basados en la investigación y experiencia en los países desarrollados [7]. En este marco, la generación Y representa a las personas que nacieron entre 1978 y 1994 [6]. Los investigadores coinciden en que los integrantes de la generación Y son conocedores de las tecnologías y están más inmersos en comportamientos de compra a través de Internet [1].

\section{Servicios de redes sociales}

La definición de SRS más utilizada en la literatura es la proporcionada por Boyd y Ellison [8]; ellos definen SRS como servicios basados en Web que permiten a los individuos (1) construir un perfil público o semipúblico dentro de un sistema limitado, (2) articular una lista de otros usuarios con los que comparten una conexión, y (3) ver y recorrer su lista de las conexiones y las hechas por otros dentro del sistema. La naturaleza y la nomenclatura de estas conexiones pueden variar de un sitio a otro.

Según Bagozzi [9], gran parte de la conducta humana se caracteriza por individuos que actúan en forma conjunta. En particular, la decisión de utilizar las tecnologías de SRS representa un fenómeno social que depende en gran medida de las interacciones entre los usuarios y el uso de las tecnologías sociales puede tener sentido sólo cuando un grupo de individuos están dispuestos a utilizar y seguir utilizando la tecnología juntos.

\section{Modelo de aceptación de tecnologías}

Propuesto por Fred Davis [10], el modelo de aceptación de la tecnología (TAM) explica el proceso de aceptación de la tecnología de la información a nivel individual. TAM postula que la intención de conducta individual de uso de la tecnología de la información queda determinada por la utilidad percibida y la facilidad de uso percibida. Al 
mismo tiempo, la facilidad de uso percibida afecta directamente a la utilidad percibida. El modelo TAM ha sido ampliamente utilizado en la literatura científica [11] y en particular en forma exitosa en contextos Internet [12]. Basados en TAM, Venkatesh y Bala [13] proponen un modelo denominado TAM 3 que amplía TAM con un conjunto de factores predictores y moderadores. TAM 3 es el última versión del modelo TAM que fue desarrollada a partir de una revisión amplia de la literatura asociada a TAM; con el mismo propósito y en forma anterior, Venkatesh, Morris y Davis habían propuesto UTAUT (Teoría unificada de aceptación y uso de tecnología) [14].

A continuación se exponen tres variables que la literatura propone como factores predictores de la aceptación y uso de tecnología en el contexto de los SNS [15]: identidad social, altruismo y telepresencia.

\section{Identidad social}

La identidad social se caracteriza por su solidaridad con el grupo social. Las personas que tienen un grado mayor de identidad social tienden a percibir sus grupos internos de manera que se distinguen positivamente de grupos externos. Estas personas prefieren pertenecer a un grupo que les proporciona una autoimagen positiva. En un SRS la identidad social es la percepción de pertenencia a la comunidad del SRS, donde la gente tiene la motivación para plantearse la interacción social con los demás [16]. La identidad social ha sido propuesta como un determinante que afecta a la intención de utilizar una determinada tecnología o sistema en comunidades virtuales [15].

\footnotetext{
Altruismo

El altruismo se puede clasificar en dos tipos: altruismo biológico y el altruismo recíproco. En el primero, el individuo beneficia a un pariente genético dándole posibilidades de supervivencia o reproducción a costo de sus propias posibilidades, en el segundo, se ayuda a los demás por la creencia de que la persona que se beneficia retornará la asistencia en el futuro [17]. En un contexto de los SRS, los usuarios pueden elaborar los dos tipos de altruismo [15]. Se ha identificado al altruismo como un factor de éxito en los procesos de producción de contenido de la Web 2.0 [18] y se ha propuesto al altruismo como un determinante que afecta las percepciones de utilidad de un SRS [15].
}

\section{Telepresencia}

La telepresencia significa que el individuo siente que se encuentra localizado en un lugar remoto del lugar donde realmente está [18]. En un ambiente de SRS la telepresencia podría incrementar la percepción de utilidad del SRS [15-16].

\section{MODELO PROPUESTO E HIPÓTESIS}

\section{Modelo}

Basado en TAM, el modelo de investigación se muestra en la Figura 1.

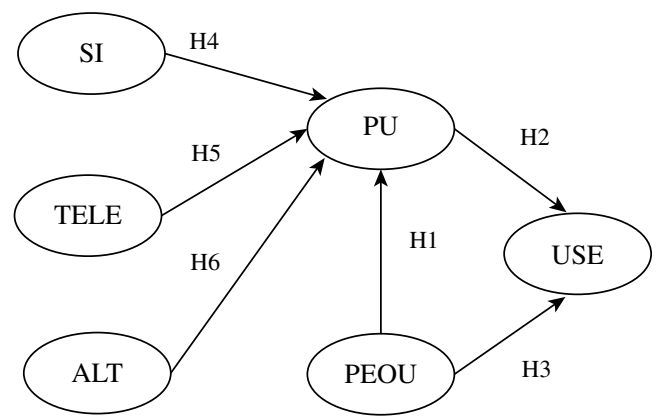

Figura 1. Modelo de investigación.

En el modelo se propone una relación entre Facilidad de Uso Percibida (PEOU) y Utilidad Percibida (PU), además se incluye el efecto de PEOU y PU sobre el Uso de SRS (USE). Este modelo TAM básico se enriquece con tres antecedentes de PU: Identidad Social (SI), Telepresencia (TELE) y Altruismo (ALT). Es decir, una mayor percepción de pertenencia a la comunidad del SRS, un comportamiento más altruista, y una mayor sensación de estar en el lugar remoto de sus contactos tienen un efecto positivo en la percepción de utilidad del SRS por parte de sus usuarios, esto último unido con una mayor percepción de la facilidad de uso del sistema, aumenta el uso de este tipo de tecnología.

\section{Hipótesis}

Basados en [10-11,13] se proponen las siguientes hipótesis:

- H1: PEOU tiene un efecto positivo sobre PU en la adopción de Servicios de Redes Sociales.

- H2: PU tiene un efecto positivo sobre USE en la adopción de Servicios de Redes Sociales.

- H3: PEOU tiene un efecto positivo sobre USE en la adopción de Servicios de Redes Sociales. 
Basados en [15-16, 18], se proponen las siguientes hipótesis:

- H4: SI tiene un efecto positivo sobre PU en la adopción de Servicios de Redes Sociales.

- H5: TELE tiene un efecto positivo sobre PU en la adopción de Servicios de Redes Sociales.

- H6: ALT tiene un efecto positivo sobre PU en la adopción de Servicios de Redes Sociales.

\section{METODOLOGÍA}

\section{Muestra}

La investigación empírica se basó en un muestreo no aleatorio de conveniencia. En particular, los datos fueron recolectados a través de un cuestionario en línea entre febrero y marzo de 2011. El cuestionario en línea fue enviado a usuarios de SRS, y estos a sus contactos. Si bien la aplicación del método "bola de nieve" no facilita la delimitación de la frontera espacial, se puede indicar que las primeras personas contactadas se ubicaron en los países de Chile (80 individuos) y España (120 individuos). A los individuos inicialmente contactados se les solicitó que pidieran a sus contactos de SRS que contestaran también el cuestionario, a cambio de ello pudieron participar en el sorteo de un artefacto electrónico, a los contactos de los contactos y a todos los siguientes contactados se les incentivó a participar en la encuesta con un segundo sorteo por otro artículo electrónico de menor valor que el primero. Los contactados iniciales fueron estudiantes y académicos universitarios, en particular, en España un grupo de los contactados iniciales eran estudiantes de intercambio. El tamaño final de la muestra fue de 771 miembros de la generación Y (individuos que nacieron entre 1978 y 1994).

\section{Escalas}

Las escalas de medición aplicadas para medir los constructos o variables latentes del modelo han sido probadas en otras investigaciones. Todos los constructos del modelo son de naturaleza reflexiva. Específicamente, para medir los constructos Percepción de Facilidad de Uso, Percepción de Utilidad y Uso se han adaptado las escalas propuestas por Venkatesh y Bala [13]. Para medir los constructos Identidad Social, Altruismo y Telepresencia se utilizaron las escalas desarrolladas por Kwon y Wen [15]. Todas las escalas utilizadas fue tipo Likert de siete puntos.

\section{RESULTADOS}

\section{Estadísticas descriptivas}

En un primer análisis se revisaron las características de género, nacionalidad y edad de la muestra, asimismo se calculó el valor medio de las variables latentes integradas en el modelo de investigación. Los resultados de las estadísticas descriptivas son mostrados en las Tablas 1, 2, 3 y 4. El software SPSS fue usado para este examen.

Tabla 1. Género de los encuestados.

\begin{tabular}{|c|c|c|}
\hline 苞 & 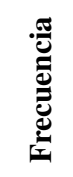 & 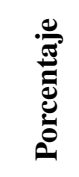 \\
\hline Masculino & 276 & 35,8 \\
\hline Femenino & 495 & 64,2 \\
\hline Total & 771 & 100,0 \\
\hline
\end{tabular}

Tabla 2. Nacionalidad de los encuestados.

\begin{tabular}{|c|c|c|c|}
\hline 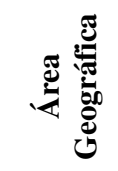 & 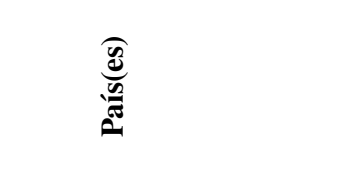 & & \\
\hline \multirow[b]{2}{*}{ Europa } & España & 486 & 63,0 \\
\hline & $\begin{array}{c}\text { Alemania, Bélgica, Francia, } \\
\text { Holanda, Italia, Polonia, } \\
\text { Rep. Checa, Rumania }\end{array}$ & 43 & 5,6 \\
\hline \multirow{3}{*}{ América } & Chile & 174 & 22,6 \\
\hline & Estados Unidos & 44 & 5,7 \\
\hline & $\begin{array}{c}\text { Argentina, Bolivia, Brasil, } \\
\text { Colombia, Cuba, Ecuador, } \\
\text { México y Perú }\end{array}$ & 20 & 2,6 \\
\hline África & Marruecos, Guinea & 4 & 0,5 \\
\hline \multicolumn{2}{|r|}{ Total } & 771 & 100,0 \\
\hline
\end{tabular}


Tabla 3. Edad de los encuestados.

\begin{tabular}{|c|c|c|}
\hline 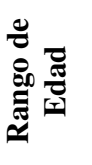 & & \\
\hline $18-21$ & 358 & 46,4 \\
\hline $22-25$ & 315 & 40,8 \\
\hline $26-29$ & 49 & 6,4 \\
\hline $30-33$ & 49 & 6,4 \\
\hline Total & 771 & 100,0 \\
\hline
\end{tabular}

Tabla 4. Valores medios de variables latentes.

\begin{tabular}{|c|c|}
\hline & \\
\hline ALT & 5,79 \\
\hline SI & 4,49 \\
\hline PEOU & 5,98 \\
\hline PU & 5,72 \\
\hline TELE & 3,90 \\
\hline USE & 4,94 \\
\hline
\end{tabular}

Acorde con la caracterización de la generación Y recogida en la literatura, en la muestra el $86 \%$ de los individuos responden que tienden a usar con frecuencia las SRS y un $70 \%$ de ellos compró productos o servicios por Internet el último año. Facebook, Tuenti y Twitter fueron los sitios de SRS más populares en la muestra. En relación a su uso, las funciones más utilizadas son las de producción de contenido e interacción: hacer comentarios $(5,13$ sobre 7), chatear (5,00 sobre 7) y mostrar fotografías (4,99 sobre 7).

\section{Valoración del modelo de investigación}

Un enfoque Partial Least Squares (PLS) para Modelos de Ecuaciones Estructurales (SEM) se usó para testar el modelo de investigación [20-21]. El software SmartPLS 2.0 de M3 se utilizó para este análisis [22].

Un modelo PLS es descrito por dos modelos: un modelo de medida entre las variables manifiestas (VM) y sus propias variables latentes (VL) y un modelo estructural entre algunas variables endógenas a otras variables.

\section{Análisis del modelo de medida}

Antes de analizar el modelo estructural, fue determinada la fiabilidad y validez del modelo de medida. La fiabilidad individual se evaluó examinado las cargas $(\lambda)$ o correlaciones simples de las medidas o indicadores con sus respectivas VL (se aceptaron los indicadores con $\lambda \geq 0,707$ ). En la Tabla 5 se muestran los resultados del procedimiento de carga cruzada de toda la muestra.

Tabla 5. Resultados del procedimiento de carga cruzada.

\begin{tabular}{|c|c|c|c|c|c|c|}
\hline 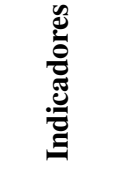 & $\frac{5}{4}$ & $\bar{n}$ & $\begin{array}{l}0 \\
0 \\
0 \\
0\end{array}$ & 2 & 受 & $\frac{\sqrt[n]{n}}{2}$ \\
\hline ALT1 & 0,87 & 0,47 & 0,29 & 0,29 & 0,28 & 0,23 \\
\hline ALT2 & 0,92 & 0,40 & 0,29 & 0,34 & 0,26 & 0,25 \\
\hline ALT3 & 0,86 & 0,28 & 0,31 & 0,31 & 0,22 & 0,20 \\
\hline SI1 & 0,31 & 0,87 & 0,20 & 0,35 & 0,30 & 0,42 \\
\hline SI2 & 0,46 & $\mathbf{0 , 9 3}$ & 0,29 & 0,40 & 0,30 & 0,45 \\
\hline SI3 & 0,40 & 0,90 & 0,19 & 0,32 & 0,31 & 0,41 \\
\hline PEOU1 & 0,30 & 0,22 & $\mathbf{0 , 8 8}$ & 0,33 & 0,11 & 0,30 \\
\hline PEOU2 & 0,28 & 0,24 & 0,88 & 0,31 & 0,14 & 0,28 \\
\hline PEOU3 & 0,32 & 0,23 & 0,91 & 0,28 & 0,09 & 0,26 \\
\hline PEOU4 & 0,24 & 0,17 & 0,74 & 0,21 & 0,04 & 0,18 \\
\hline PU1 & 0,25 & 0,32 & 0,22 & 0,81 & 0,37 & 0,50 \\
\hline PU2 & 0,27 & 0,35 & 0,23 & $\mathbf{0 , 8 3}$ & 0,35 & 0,46 \\
\hline PU3 & 0,34 & 0,29 & 0,35 & 0,84 & 0,27 & 0,44 \\
\hline PU4 & 0,32 & 0,34 & 0,31 & 0,80 & 0,26 & 0,43 \\
\hline TELE1 & 0,28 & 0,33 & 0,10 & 0,37 & 0,84 & 0,34 \\
\hline TELE2 & 0,27 & 0,24 & 0,13 & 0,31 & 0,84 & 0,27 \\
\hline TELE3 & 0,09 & 0,21 & 0,03 & 0,20 & $\mathbf{0 , 7 3}$ & 0,18 \\
\hline USE1 & 0,28 & 0,35 & 0,34 & 0,56 & 0,26 & $\mathbf{0 , 8 9}$ \\
\hline USE2 & 0,20 & 0,41 & 0,24 & 0,46 & 0,32 & 0,92 \\
\hline USE3 & 0,20 & 0,53 & 0,23 & 0,47 & 0,35 & $\mathbf{0 , 8 8}$ \\
\hline
\end{tabular}


La fiabilidad de la VL indica la rigurosidad con que las variables observadas miden la misma VL; el coeficiente $\alpha$ de Cronbach fue utilizado como el índice de fiabilidad de las VL (fueron aceptadas VL con $\alpha>0,7)$. Además, la fiabilidad compuesta fue calculada. La validez convergente de la VL se evaluó mediante el examen de la varianza media extraída (AVE), según Fornell y Larcker [23] (se aceptaron AVE $>0,5$ ). La Tabla 6 muestra el coeficiente $\alpha$ de Cronbach, AVE y fiabilidad compuesta por cada VL.

Tabla 6. $\alpha$ de Cronbach, AVE y fiabilidad compuesta.

\begin{tabular}{|c|c|c|c|}
\hline 总 & $\underset{\circlearrowright}{\circlearrowright}$ & $\frac{1}{4}$ & 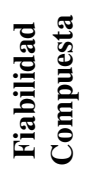 \\
\hline ALT & 0,86 & 0,78 & 0,91 \\
\hline SI & 0,89 & 0,81 & 0,93 \\
\hline PEOU & 0,88 & 0,73 & 0,91 \\
\hline PU & 0,84 & 0,67 & 0,89 \\
\hline TELE & 0,74 & 0,65 & 0,85 \\
\hline USE & 0,88 & 0,80 & 0,92 \\
\hline
\end{tabular}

Se probó la validez discriminante de las VL analizando si la raíz cuadrada del AVE de cada LV es mayor que las correlaciones con el resto de las LV (véase Tabla 7).

Tabla 7. Correlaciones de VL para toda la muestra (elementos en la diagonal son las raíces cuadradas del AVE).

\begin{tabular}{|c|c|c|c|c|c|c|}
\hline & 㝵 & $\tilde{n}$ & $\begin{array}{l}8 \\
0 \\
2 \\
2\end{array}$ & 2 & 结 & $\begin{array}{l}\frac{1}{2} \\
\stackrel{2}{\Omega}\end{array}$ \\
\hline ALT & $\mathbf{0 , 8 8}$ & - & - & - & - & - \\
\hline SI & 0,43 & 0,90 & - & - & - & - \\
\hline PEOU & 0,34 & 0,25 & $\mathbf{0 , 8 5}$ & - & - & - \\
\hline $\mathbf{P U}$ & 0,36 & 0,40 & 0,34 & $\mathbf{0 , 8 2}$ & - & - \\
\hline TELE & 0,29 & 0,33 & 0,12 & 0,38 & $\mathbf{0 , 8 1}$ & - \\
\hline USE & 0,26 & 0,47 & 0,30 & 0,56 & 0,34 & $\mathbf{0 , 9 0}$ \\
\hline
\end{tabular}

\section{Análisis del modelo estructural}

Después de comprobar validez y fiabilidad del modelo de medida, fueron testeadas las relaciones entre los constructos. Las hipótesis del estudio fueron evaluadas mediante el examen de los coeficientes de camino $(\beta)$ y sus niveles de significación. Un bootstrapping con 500 submuestras fue realizado para comprobar la significación estadística de cada uno de los coeficientes de los caminos. La varianza explicada (R-cuadrado) en las VL endógenas y el coeficiente de significación de regresión (F-test) sirven como indicadores de la capacidad explicativa del modelo. El GoF calculado con un valor de 0,48 provee un adecuado soporte a la validez global del modelo [24]. La Figura 2 muestra el resultado del análisis PLS.

Con base a estos resultados, las hipótesis $\mathrm{H} 1, \mathrm{H} 2$, H3, H4, H5 y H6 del estudio no se rechazan.

Estos hallazgos indican que en una muestra amplia de individuos pertenecientes a la generación $\mathrm{Y}$ existe una fuerte y positiva relación $(0,51)$ entre la percepción de utilidad de los servicios de redes sociales y su uso, además este uso está influenciado en menor forma $(0,13)$ por la percepción de la facilidad de su uso. También, cuando aumenta esta percepción de facilidad de uso, se incrementa $(0,22)$ la percepción de utilidad de los servicios de redes sociales. Por otra parte, la percepción de utilidad de los servicios de redes sociales está determinada parcialmente $\left(R^{2}=0,29\right)$ por el nivel de telepresencia $(0,25)$, identidad social $(0,20)$ y altruismo $(0,13)$ de los usuarios.

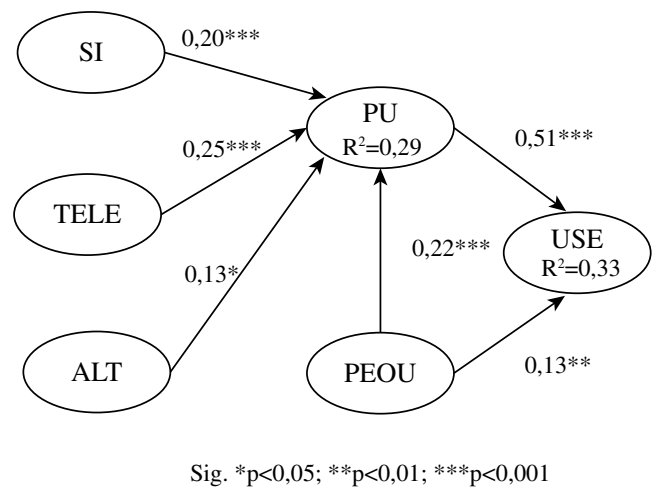

Figura 2. R-cuadrado y camino estimado del análisis PLS. 


\section{CONCLUSIONES, LIMITACIONES Y ESTUDIOS FUTUROS}

Como conclusión queremos destacar tres contribuciones de este estudio. Primero, se ha podido utilizar exitosamente el modelo basado en TAM para explicar el proceso de adopción SRS en una amplia muestra de encuestados de diversos países pertenecientes a la generación Y (se comprobó la validez, fiabilidad y capacidad explicativa de modelo propuesto). Segundo, un hallazgo importante es la comprobación de una significativa relación en este grupo generacional entre telepresencia y utilidad perciba, lo que tiene implicaciones para el diseño de estos servicios (a mayor percepción de telepresencia mayor percepción de utilidad y mayor uso del SRS). Este efecto significativo entre telepresencia y utilidad percibida puede interpretarse como una indicación de que los usuarios de estos servicios prefieren interactividad y riqueza de medios en estas tecnologías. Y tercero, fue posible encontrar una relación significativa entre identidad social y utilidad percibida, lo que explicita una característica actitudinal de la generación Y al utilizar Web 2.0. este efecto significativo entre identidad social y utilidad percibida indica una característica de los miembros de la generación Y, si ellos conciben el uso de SRS como una forma de pertenencia a una comunidad donde las personas se sienten motivadas a interactuar socialmente con otras, mostrarán una mayor percepción de utilidad de estas tecnologías.

Finalmente, podemos indicar una implicación de este estudio. La generación Y es solidaria, los miembros de la generación Y creen que su contribución a la sociedad es importante. Los resultados del estudio apoyan esta idea en el contexto de SRS, el altruismo y la identidad social (que incluye la solidaridad del grupo) son las variables exógenas más valoradas por los encuestados. Creemos que este sentido de la solidaridad puede ser una clave importante para explorar en futuros estudios sobre el comportamiento de esta generación.

Este estudio posee dos importantes limitaciones que orientan los futuros trabajos relacionados. Primero, el tipo de muestreo voluntario es una limitación para la generalización de los hallazgos. Y segundo, el estudio es transversal, sería aconsejable un estudio longitudinal para comparar las distintas etapas temporales de la adopción SRS.
Este trabajo es el inicio de una serie de estudios que se orientarán a determinar si existen al interior de la muestra diferencias significativas entre grupos de distintos géneros, nacionalidades o comportamientos de compra.

\section{AGRADECIMIENTOS}

Se agradece el apoyo financiero del grupo de investigación SEJ494: E-Business: Empresa, Administración y Ciudadano, de la Universidad de Sevilla, España.

\section{REFERENCIAS}

[1] K. Nusair, H.G. Parsa and C. Cobanoglu. "Building a model of commitment for Generation Y: An empirical study on e-travel retailers". Tourism Management. Vol. 32, Issue 4, pp. 833-843. 2011.

[2] G. Ritzer and N. Jurgenson. "Production, Consumption, Prosumption: The nature of capitalism in the age of the digital 'prosumer'". Journal of Consumer Culture. Vol. 10, Issue 1, pp. 13-36. 2010.

[3] Nielsen Wire. "Social Networks/Blogs Now Account for One in Every Four and a Half Minutes Online". June 15, 2010. Date of visit: May 30, 2011. URL: http://blog. nielsen.com/nielsenwire/online_mobile/ social-media-accounts-for-22-percent-oftime-online/.

[4] Facebook. "Statistics". May 30, 2011. Date of visit: May 30, 2011. URL: http://www. facebook.com/press/info.php?statistics.

[5] M. Trusov, A.V. Bodapati and R.E. Bucklin. "Determining Influential Users in Internet Social Networks". Journal of Marketing Research. Vol. 47, Issue 4, pp. 643-658. 2010.

[6] P. Sheahan. "Generation Y: Thriving and surviving with generation $Y$ at work". Hardie Grant Books, New York, USA. 2009.

[7] T. Baum. "Tourism and Generation Y". Tourism Management. Vol. 32, Issue 4, pp. 960-961. 2011.

[8] D.M. Boyd and N.B. Ellison. "Social network sites: Definition, history, and scholarship". Journal of Computer-Mediated Communication. Vol. 13, Issue 1, pp. 210213. 2007. 
[9] R.P. Bagozzi. "The legacy of the technology acceptance model and a proposal for a paradigm shift". Journal of the Association of Information Systems. Vol. 8, Issue 4, pp. 244-254. 2007.

[10] F.D. Davis. "Perceived usefulness, perceived ease of use, and user acceptance of information technologies”. MIS Quarterly. Vol. 13, Issue 3, pp. 319-340. 1989.

[11] V. Venkatesh and M.G. Morris. "Why don't men ever stop to ask for directions? Gender, social influence, and their role in technology acceptance and usage behavior". MIS Quarterly. Vol. 24, Issue 1, pp. 115-139. 2000.

[12] J. Arenas-Gaitán, P. Ramírez-Correa and F.J. Rondan-Cataluña. "Cross cultural analysis of the use and perceptions of Web based learning systems". Computers \& Education. Vol. 57, Issue 2, pp. 1762-1774. 2011.

[13] V. Venkatesh and H. Bala. "Technology Acceptance Model 3 and a Research Agenda on Interventions". Decision Sciences. Vol. 39, Issue 2, pp. 273-315. 2008.

[14] V. Venkatesh, M.G. Morris and G.B. Davis. "User Acceptance of Information Technology: Toward a Unified View”. MIS Quarterly, Vol. 27, Issue 3, pp. 425-478. 2003.

[15] O. Kwon and Y. Wen. "An empirical study of the factors affecting social network service use". Computers in Human Behavior. Vol. 26, Issue 2, pp. 254-263. 2010.

[16] P. Sombutpiboo. "The Adoption of Social Networks in Thailand". Journal of Information Technology Impact. Vol. 11, Issue 1, pp. 1-34. 2011.
[17] R.L. Trivers. "The evolution of reciprocal altruism". Quarterly Review of Biology. Vol. 46, Issue 1, pp. 35-57. 1971.

[18] P. Prasarnphanich and C. Wagner. "The role of Wiki technology and altruism in collaborative knowledge creation". Journal of Computer Information Systems. Vol. 49, Issue 4, pp. 33-41. 2009.

[19] T. Kim and F. Biocca. "Telepresence via television: Two dimensions of telepresence may have different connections to memory and persuasion". Journal of ComputerMediated Communication, Vol. 3, Issue 2. 2004.

[20] W.W. Chin. "The partial least squares approach for structural equation modeling". In G.A. Marcoulides (Ed.), Modern Methods for Business Research. Lawrence Erlbaum Associates. USA. 1998.

[21] M. Tenenhaus, V.E. Vinzi, Y-M. Chatelin and C. Lauro. "PLS path modeling". Computational Statistics and Data Analysis. Vol. 48, pp. 159-205. 2005.

[22] C.M. Ringle, S. Wende and A. Will. "SmartPLS 2.0 (M3) beta". Hamburg. 2005. URL: http://www.smartpls.de

[23] C. Fornell and D.F. Larcker. "Evaluating structural equation models with unobservable variables and measurement error". Journal of Marketing Research. Vol. 18, Issue 1, pp. 39-50. 1981.

[24] M. Wetzels, G.O. Schroder and V.C. Oppen. "Using PLS path modeling for assessing hierarchical construct models: Guidelines and empirical illustration”. MIS Quarterly, Vol. 33, Issue 1, pp. 177-195. 2009. 